\title{
Azithromycin: assessment of intrinsic cytotoxic effects on corneal epithelial cell cultures
}

This article was published in the following Dove Press journal:

Clinical Ophthalmology

23 May 2013

Number of times this article has been viewed

\section{Rita Mencucci' \\ Domenico E Pellegrini- \\ Giampietro² \\ lacopo Paladini' \\ Eleonora Favuzza' \\ Ugo Menchini' \\ Tania Scartabelli²}

'Department of Specialized Surgical Science - Eye Clinic, ${ }^{2}$ Department

of Health Science Section of Clinical Pharmacology and Oncology,

University of Florence, Florence, Italy
Correspondence: Rita Mencucci Department of Specialized Surgical Sciences - Eye Clinic, University of Florence, Largo Brambilla 3, 50134 Florence, Italy Email rita.mencucci@unifi.it
Purpose: To compare the cytotoxic effects of preservative-free azithromycin on corneal epithelial cells in vivo with those of preservative-free netilmicin and levofloxacin, and the preservative benzalkonium chloride (BAK).

Methods: Rabbit corneal epithelial cells in vitro were incubated for 15 minutes or 6 hours with commercially available ophthalmic preservative-free netilmicin $0.3 \%$, levofloxacin $0.3 \%$, or azithromycin $1.5 \%$ preparations or different concentrations of unpreserved azithromycin and different concentrations of BAK. Qualitative analysis was undertaken using phase-contrast optics to examine the morphological aspects of cell cultures and quantitative analysis was undertaken by measuring the release of the cytoplasmic enzyme lactate dehydrogenase into the medium immediately and 24 hours after exposure to drugs. Finally, we observed the wound-healing rate of mechanically injured corneal epithelial cells exposed to each antibiotic ophthalmic preparation for 48 hours.

Results: Our results show that both the commercially available unpreserved mono-dose preparation of azithromycin and ophthalmic preparations of azithromycin up to a concentration of $1.5 \%$ were virtually devoid of harmful effects under our experimental conditions. This was not significantly different from the results obtained for the other antibiotic preparations $(P>0.05)$ tested, but was unlike the results obtained for BAK. Azithromycin 1.5\% also showed good recovery properties after a mechanical wound test.

Conclusion: Under our experimental conditions, unpreserved azithromycin $1.5 \%$ showed a much lower toxicity than BAK and did not interfere with the wound-healing process.

Keywords: macrolides, toxicity, corneal epithelial cell culture, wound healing, preservative, benzalkonium chloride

\section{Introduction}

Azithromycin is an azalide, a second-generation 15-C-atom macrolide antibiotic with broad-spectrum activity against Gram-positive, Gram-negative, and atypical bacteria such as Chlamydia trachomatis. It shows a potency higher than erythromycin against Haemophilus influenzae and Neisseria gonorrhoeae in vitro. ${ }^{1,2}$

Azithromycin has an intracellular action, as it binds to the bacterial ribosomal subunit $50 \mathrm{~S}$ and inhibits microbial protein synthesis. It is also rapidly distributed in the tissue and has good intracellular penetration and transport into phagocytic cells, reaching high and sustained levels in tissue - especially at infection sites - including in ocular tissue. Thanks to its optimal pharmacokinetic profile, this antibiotic exhibits good bacteriostatic and bactericidal time-dependent action and a long post-antibiotic effect, thus permitting a short oral or topical 3-day administration regimen. ${ }^{3-7}$ 
In vitro studies have reported that azithromycin has anti-inflammatory and immunomodulatory actions in addition to its antimicrobial activity, such as the inhibition of cytokine and proinflammatory mediator synthesis and inflammatory cell migration, and the suppression of the nuclear factor kappa B signaling transduction pathway, ${ }^{8-12}$ thus providing a rationale for clinical investigation into the off-label use of azithromycin eye drops in chronic blepharitis. ${ }^{1}$

Azithromycin $1.5 \%$ ophthalmic solution is approved in several countries in the European Union for the topical treatment of purulent bacterial conjunctivitis caused by susceptible strains and of trachomatous conjunctivitis caused by Chlamydia trachomatis. ${ }^{1,13-16}$ Azithromycin $1.5 \%$ ophthalmic solution proved effective and was well tolerated in patients with these infections. ${ }^{14,15}$ Two welldesigned studies showed that in terms of clinical cure rates, treatment with azithromycin $1.5 \%$ ophthalmic solution for 3 days was non-inferior to treatment with tobramycin $0.3 \%$ ophthalmic solution for 7 days in pediatric and adult patients with purulent bacterial conjunctivitis and non-inferior to a single dose of azithromycin oral suspension in pediatric patients with trachomatous conjunctivitis. ${ }^{14,15}$ Moreover, an azithromycin $1.5 \%$ ophthalmic solution treatment regimen is shorter and has less frequent dosing requirements than a tobramycin treatment regimen (potentially improving compliance), and has been associated with quicker resolution of clinical signs of bacterial conjunctivitis..$^{14,15}$

The most common adverse events listed in the summary of product characteristics as occurring during clinical trials and from post-marketing safety data are related to ocular discomfort (ie, pruritus, burning, and stinging) on instillation. Less common adverse events include blurred vision, sticky eye sensation, and foreign body sensation on instillation. To the best of our knowledge, no in vitro studies on the toxicity of an azithromycin ophthalmic solution on corneal epithelial cells has been reported. However, Wingard et al performed an azithromycin toxicity assay on corneal epithelial cells as part of an extensive study designed to investigate the ability of some antibiotics to protect mammalian cells from destruction by bacteria, and showed that azithromycin had low in vitro toxicity. ${ }^{17}$

The aim of this in vitro study was to evaluate the cytotoxic effects of a commercially available azithromycin $1.5 \%$ ophthalmic solution on rabbit corneal epithelial cells and to compare these effects with those of other commonly used antibiotic eye drops. Benzalkonium chloride (BAK) was used as positive control. BAK is a quaternary ammonium compound used as a preservative in over $70 \%$ of the existing multi-dose ophthalmic solution bottles, at an average concentration of $0.01 \%$. BAK is known to promote activation of lipoxygenases and the synthesis and secretion of eicosanoids, inflammatory mediators, and many cytokines such as interleukin (IL)- $1 \alpha$, IL-8, IL-10, and tumor necrosis factor-alpha, resulting in delayed hypersensitivity and allergic reactions. ${ }^{18}$

Three mechanisms of BAK toxicity have been described: (1) a detergent effect causing loss of tear-film stability, (2) direct damage to the corneal and conjunctival epithelium, and (3) an immunoallergic reaction. It acts by denaturing proteins and disrupting cytoplasmic membranes. ${ }^{18}$

\section{Materials and methods Incubation with drugs}

Statens Serum Institut rabbit corneal (SIRC) cells were purchased from Sigma-Aldrich (St Louis, MO, USA). Cells were resuspended and seeded into 24-well plates

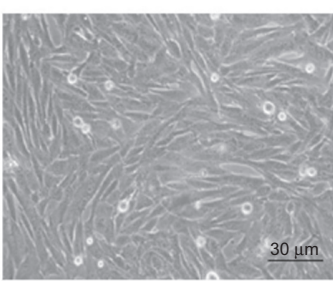

$\mathrm{NaCl} 0.09 \%$

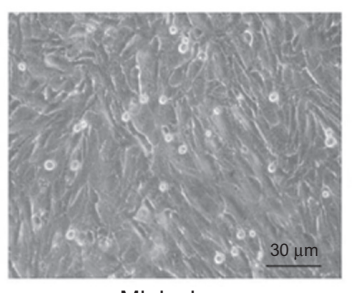

Miglyol

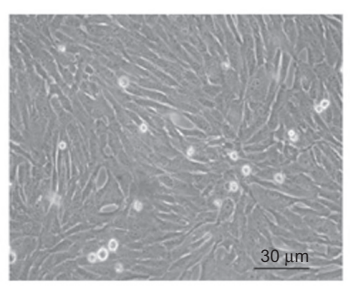

Oftaquix

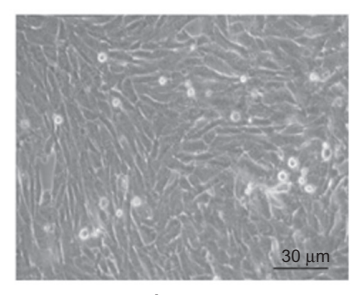

Azyter

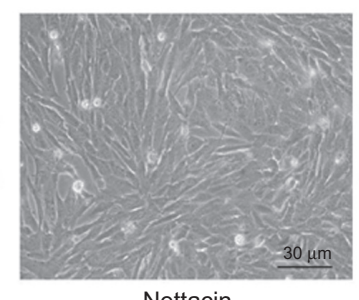

Nettacin

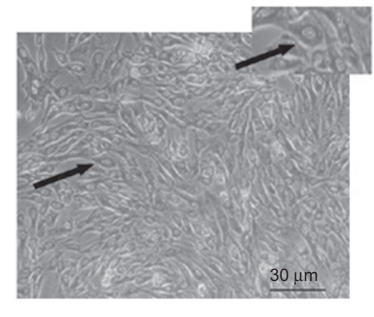

$0.01 \%$ BAK
Figure I Phase-contrast micrographs of untreated (control) and benzalkonium chloride (BAK)-treated cultured rabbit corneal epithelial cells.

Notes: Control cultures display normal cell morphology with long processes, no cytoplasmatic granularity, and multiple intracellular contacts. Incubation with BAK $(0.005 \%-0.010 \%)$ for 15 minutes resulted in a dose-dependent cytotoxicity; cells show "rounding" of the cell bodies, thickening of cellular processes, and vacuolization of the cytoplasm (arrows). Incubation with azithromycin 1.5\% (Azyter; Laboratoires Théa, Clermont-Ferrand, France), Miglyo ${ }^{\circledR}$ (Laboratoires Théa), netilmicin 0.3\% (Nettacin ${ }^{\circledR}$; Società Industria Farmaceutica Italiana, Catania, Italy) or levofloxacin $0.3 \%$ (Oftaquix; Bausch and Lomb, Milan, Italy) (containing no BAK) resulted in negligible signs of toxicity, in a manner that was similar to what was observed in control cultures treated with saline. 
Table I Assessment of severity of morphologic criteria

\begin{tabular}{lllll}
\hline & $\begin{array}{l}\text { [c] active } \\
\text { principle }\end{array}$ & [c] BAK & 15 minutes & 6 hours \\
\hline Control $^{\text {Azyter }}$ & 0 & 0 & 0 & 0 \\
Miglyol $^{\circledR b}$ & $1.5 \%$ & 0 & 0 & 0 \\
Nettacin $^{\circledR c}$ & $0.3 \%$ & 0 & 0 & 0 \\
Oftaquix $^{d}$ & $0.3 \%$ & 0 & 0 & 0 \\
BAK & 0 & $0.0025 \%$ & 0 & 0 \\
BAK & 0 & $0.005 \%$ & + & + \\
BAK & 0 & $0.01 \%$ & ++ & ++ \\
\hline
\end{tabular}

Notes: Using phase-contrast microscopy, severity of morphologic criteria ("rounding" of cells, loss of processes, cytoplasmic granularity, and isolation and detachment of cells) were assessed semiquantitatively: 0 , none (ie, spindle-shaped cells, multiple long delicate processes, no cytoplasmic granularity, multiple intercellular contacts); +, mild (ie, spindle-shaped cells, short thickened processes in less than one-third of the cells, cytoplasmic granules detectable in less than one-third of the cells, reduced intercellular contacts); ++, moderate (ie, triangular- or polygonal-shaped cells, short thickened processes in more than one-third of the cells, cytoplasmic granules in more than one-third of cells, few intercellular contacts, moderate cell detachment); ++, severe (ie, round cells, no processes, no cell contact, severe cell detachment). ${ }^{\text {AAzithromycin }}$ I.5\% (Azyter; Laboratoires Théa, Clermont-Ferrand, France); 'Laboratoires Théa ${ }^{c_{n}}$ etilmicin $0.3 \%$ (Nettacin ${ }^{\circledR}$; Società Industria Farmaceutica Italiana, Catania, Italy); levofloxacin 0.3\% (Oftaquix; Bausch and Lomb, Milan, Italy).

Abbreviation: BAK, benzalkonium chloride; [c], concentration.

(approximately $4 \times 10^{-4}$ cells $/ \mathrm{cm}^{2}$ ) using a medium containing 90\% Eagle's minimal essential medium, 10\% heat-inactivated fetal bovine serum, and $1 \mathrm{mmol} / \mathrm{L}$ glutamine. When they reached approximately $70 \%-80 \%$ confluence, the culture medium was removed and cells were exposed to one of the following preservative-free commercially available ophthalmic preparations: azithromycin 1.5\% (Azyter; Laboratoires Théa, Clermont-Ferrand, France); caprylic/capric acid triglyceride (Miglyol ${ }^{\circledR}$, Laboratoires Théa), a vehicle of Azyter, a medium-chain-length triglyceride of saturated fatty acids (Laboratoires Théa); netilmicin $0.3 \%\left(\right.$ Nettacin $^{\circledR}$, Società Industria Farmaceutica Italiana, Catania, Italy); and levofloxacin $0.3 \%$ (Oftaquix, Bausch and Lomb, Milan, Italy). Three different concentrations of BAK $(0.0025 \%, 0.0050 \%$, and $0.0100 \%$ ) were used as toxicity controls, whereas $0.9 \%$ $\mathrm{NaCl}$ was used as negative control. Moreover, azithromycin solution (the active principle of Azyter) was applied in five different concentrations ranging from $0.5 \%$ to $2.5 \%$.

SIRC cells were incubated with drugs according to three different protocols: (1) short-term (ie, 15 minutes) exposure, (2) short-term exposure followed by the addition of drug-free medium for a 24-hour recovery period, and (3) long-term (ie, 6 hours) exposure.

\section{Phase-contrast microscopy}

After incubation with drugs according to the three experimental protocols, the SIRC cells were evaluated qualitatively in a blind fashion and photographed by an inverted phase-contrast microscope (Olympus IX-50; Olympus Europa Holding GmbH, Hamburg, Germany). Morphologic criteria indicating cytotoxic drug effects - "rounding" of cells, loss of processes, increased granularity, isolation of cells, vacuolization, and cell detachment - were assessed semiquantitatively according to criteria originally described by Seitz et al, ${ }^{19}$ with some modifications: cellular morphology (normal, 0; slight alterations, +; altered, ++), cellular boundaries (not recognizable, 0 ; mixed shapes, + ; good definition, ++), nucleus and cytoplasm (normal, 0; slight alterations, +; altered, ++), nuclear morphology (well-spread chromatin, 0 ; mixed shapes, + ; altered [pycnosis, cariorexis, etc], ++), and vacuolization (absent, 0 ; rare vacuoles, +; vacuolization present, ++ ).

\section{Lactate dehydrogenase (LDH) assay}

Cell damage in SIRC cells was quantitatively evaluated by measuring the amount of the soluble cytosolic enzyme LDH released from injured cells into the extracellular fluid immediately or 24 hours after exposure to drugs, as previously described for neurons ${ }^{20}$ or corneal fibroblasts. ${ }^{21}$

The LDH level corresponding to complete cell death was determined for each experiment by assaying sister cultures exposed to BAK $(0.0025 \%-0.0100 \%)$ for the same incubation period used for experimental drugs. Background LDH release was determined in control cultures not exposed to drugs and was subtracted from all experimental values. The resulting values correlated linearly with the degree of cell loss estimated by observation of cultures under phase-contrast optics or under bright-field optics following 5 minutes incubation with $0.4 \%$ trypan blue, which stains debris and nonviable cells. All experiments were carried out in triplicate. The activity of LDH was quantified using a Cytotoxicity Detection Kit (LDH) from Roche Diagnostics (Basel, Switzerland).

\section{Analysis of corneal wound healing}

SIRC cell cultures were wounded using a small scalpel in 24-well culture plates. ${ }^{22}$ The injured SIRC cell cultures were incubated with Azyter, Miglyol, $\mathrm{NaCl}$, or BAK for 24 or 48 hours. Three different experiments ( $\mathrm{n}=6$ per group) were undertaken. The extent of healing was evaluated qualitatively in a blind fashion and photographed by an inverted phasecontrast microscope (Olympus IX-50) at 0 and 48 hours. To quantify wound areas, they were identified and encompassed in a frame using Image-Pro Plus morphometric analysis software (version 5.0 Media Cybernetics, Rockville, MD, USA) and the extent of healing was evaluated quantitatively by 
A

15 min exposure

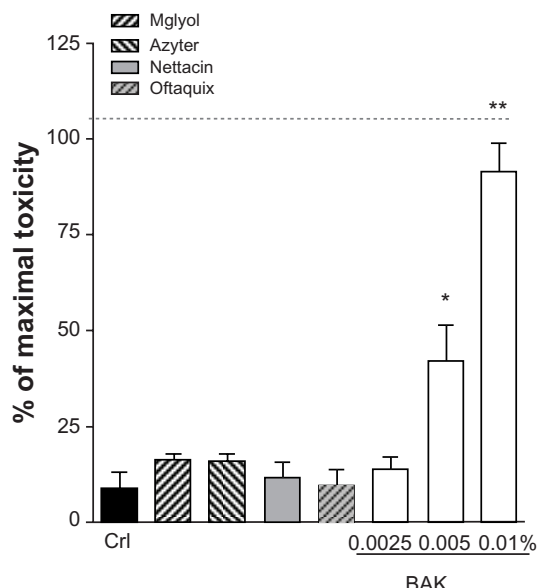

B

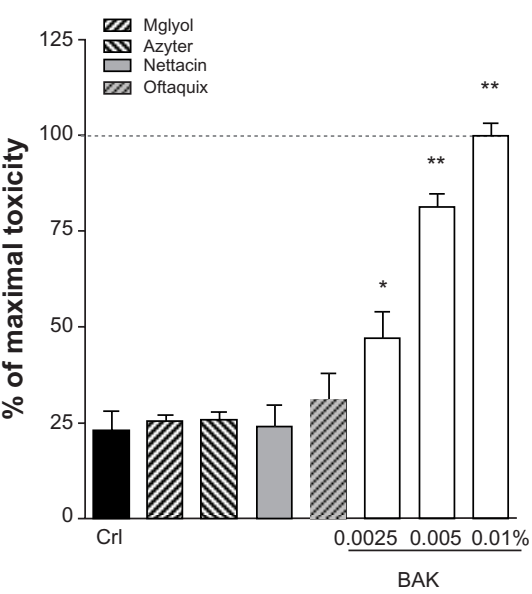

C

15 min exposure +

$24 \mathrm{~h}$ recovery

Figure 2 Evaluation of lactate dehydrogenase (LDH) release in Statens Serum Institut rabbit corneal (SIRC) cell cultures after (A) I5 minutes or (B) 6 hours of incubation with commercially available ophthalmic preparations of azithromycin (Azyter; Laboratoires Théa, Clermont-Ferrand, France), netilmicin (Nettacin ${ }^{\circledR}$; Società Industria Farmaceutica Italiana, Catania, Italy), and levofloxacin (Oftaquix; Bausch and Lomb, Milan, Italy) and benzalkonium chloride (BAK; 0.0025\%-0.0100\%). (C) Evaluation of LDH release in SIRC cell cultures 24 hours after 15 minutes of incubation with all compounds.

Notes: Data are expressed as percentage of the maximal degree of cell death (incubation of cells for 6 hours with $0.01 \%$ of BAK); they represent the mean \pm standard error of the mean of at least three experiments performed in triplicate. Statistical analysis was performed using a one-way analysis of variance and Dunnett's post-hoc test $(* P \leq 0.05$ vs control $[\mathrm{crl}] ; * * P \leq 0.01$ vs $\mathrm{crl})$.

determining the ratio of the difference between the wound areas at time 0 and remaining wound areas after 48 hours.

\section{Statistical analysis}

Data are presented here as mean \pm standard error of the mean of $n$ experiments. The statistical significance of differences for the results displayed in Figures 2-5, and 7 was analyzed using a one-way analysis of variance and Dunnett's post-hoc test. A $P$ value of 0.05 was considered significant. All statistical calculations were performed using GraphPad Prism (v 4; GraphPad Software, San Diego, CA, USA).

\section{Results}

\section{Phase-contrast microscopy}

SIRC cell cultures examined by phase-contrast microscopy following their incubation with Azyter, Miglyol, Nettacin, or Oftaquix demonstrated a pattern of cell toxicity that was negligible and comparable to that observed in control cultures (Figure 1 and Table 1).

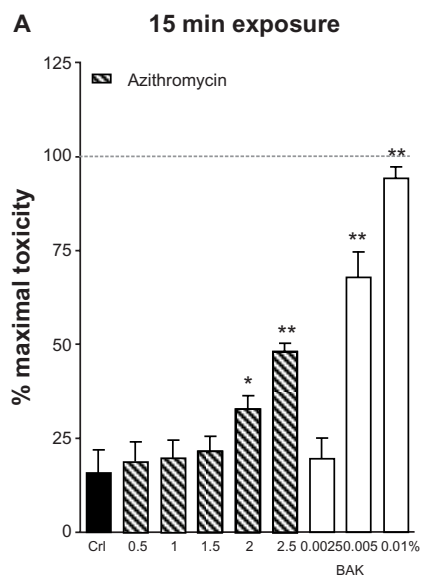

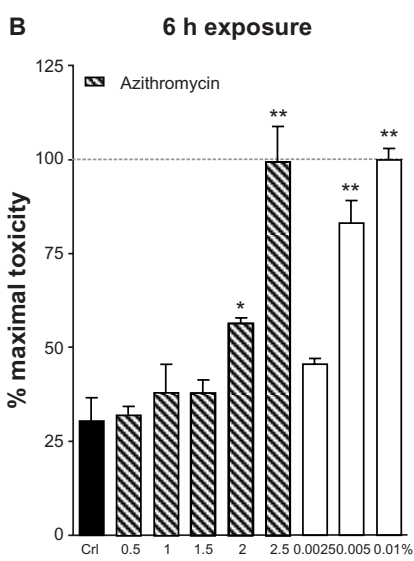

BAK

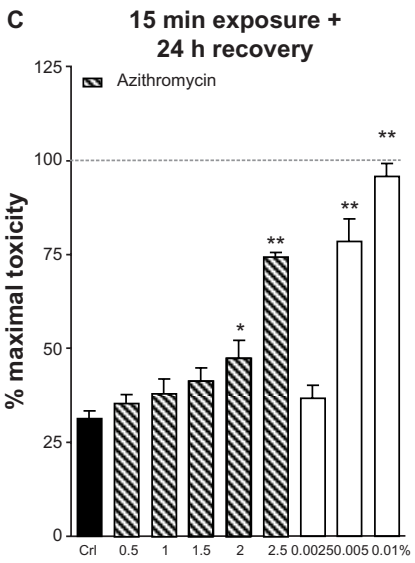

BAK

Figure 3 Evaluation of lactate dehydrogenase (LDH) release in Statens Serum Institut rabbit corneal (SIRC) cell cultures after (A) I5 minutes or (B) 6 hours of incubation with azithromycin $0.5 \%-2.5 \%$. (C) Evaluation of LDH release in SIRC cultures 24 hours after 15 minutes incubation with all different concentrations of azithromycin. Notes: Data are expressed as percentage of the maximal degree of cell death (incubation of cells for 6 hours with $0.01 \%$ of benzalkonium chloride [BAK]); they represent the mean \pm standard error of the mean of at least three experiments performed in triplicate. Statistical analysis was performed using a one-way analysis of variance and Dunnett's post-hoc test $(* P \leq 0.05$ vs control [crl]; $* * P \leq 0.0$ l vs crl). 
In contrast, the addition of BAK to the incubation medium resulted in a time- and dose-dependent increase in the appearance of cytotoxicity signs, including rounding of the cell bodies, loss of processes, and vacuolization of the cytoplasm, which eventually led to cell detachment and death (Figure 1 and Table 1).

\section{LDH assay}

Using LDH as a quantitative cytotoxicity index, we examined the extent of SIRC cell death (Figure 2). The maximal degree of cell death was produced in our culture system by incubation for 6 hours with $0.01 \%$ BAK, which produced a release of $\mathrm{LDH}$ (115 units/L) that was approximately threefold higher than the basal levels detected at the same time point (32 units/L) (Figure 2B). Incubation with $0.01 \%$ BAK for only 15 minutes produced a similar increase in LDH release (105 units/L) that was sixfold higher than the basal levels at the same time point (16 units/L) (Figure 2A). Incubation with $0.0025 \%-0.0050 \%$ BAK evoked a similar time-dependent but less pronounced increase in the release of LDH from SIRC cell cultures (Figure 2).

After 15 minutes of incubation, all compounds displayed negligible SIRC cell death (Figure 2A). A similar pattern supporting the nontoxic effects of Azyter and Miglyol was observed after 6 hours (Figure 2B). To examine whether these compounds could exert their toxic effects at more distant time intervals following exposure, we exposed SIRC cell cultures to all compounds for 15 minutes and measured LDH release 24 hours later (Figure 2C). Our results showed that Azyter was devoid of toxicity even when cell death was assessed at 24 hours after its application to SIRC cell cultures.

Finally, we tested different concentrations of azithromycin $(0.5 \%-2.5 \%)$ according to our three different protocols (Figure 3). Azithromycin displayed no signs of toxicity at concentrations below $2.0 \%$ but displayed signs of toxicity at $2.0 \%$ and $2.5 \%$ that were comparable to those displayed by BAK $0.005 \%$ (after 15 minutes of exposure) or $0.01 \%$ (after 6 hours of exposure).

\section{Corneal wound healing}

In control SIRC cell cultures $(\mathrm{NaCl} 0.9 \%)$, analysis of the corneal wound surface revealed that the wound was almost completely covered and repaired after 48 hours (87.5\%). Treatment with Azyter or Miglyol for 48 hours somewhat reduced wound healing $(47.3 \%$ and $25.6 \%$, respectively), whereas incubation with $0.01 \%$ BAK for 48 hours severely inhibited epithelial wound healing (-36\%) (Figures 4 and 5).

\section{Discussion}

While the safety of azithromycin ophthalmic solutions has been largely studied in vivo in rabbits, to our knowledge, this is the first in vitro study on the toxicity of an azithromycin ophthalmic solution in corneal epithelial cells. The aim of our study was to evaluate the cytotoxic effects of a commercially available azithromycin $1.5 \%$ ophthalmic solution in rabbit corneal epithelial cells and to compare these with those of other commonly used antibiotic eye drops.

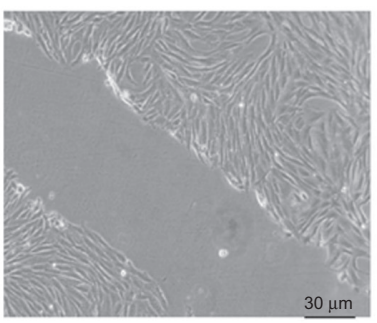

$\mathrm{NaCl} 0.09 \%$

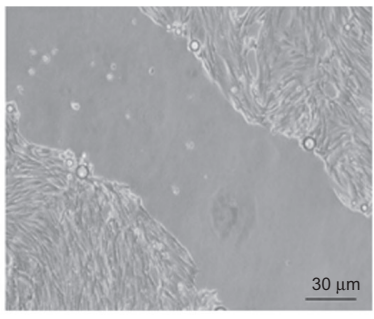

Azyter

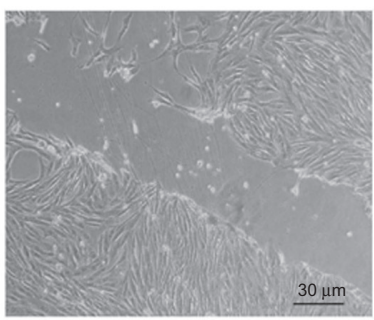

Miglyol

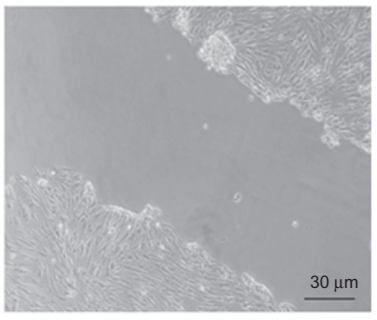

BAK $0.01 \%$

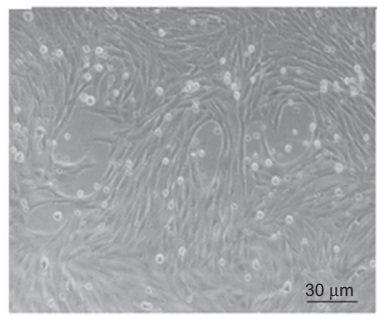

$\mathrm{NaCl} 0.9 \% 48 \mathrm{~h}$

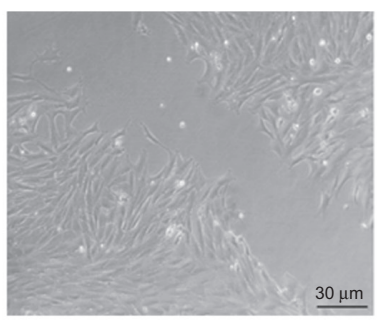

Azyter $48 \mathrm{~h}$

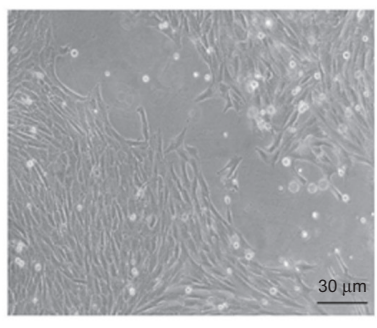

Miglyol $48 \mathrm{~h}$

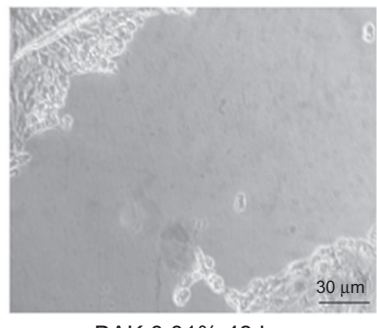

BAK $0.01 \% 48 \mathrm{~h}$
Figure 4 Phase-contrast micrographs of wounded cultured Statens Serum Institut rabbit corneal (SIRC) cells.

Notes: Left column shows the SIRC cells as wounded by a small scalpel in a culture dish. The wound of the control (top right) was healed after 48 hours. In cultures treated with azithromycin 1.5\% (Azyter; Laboratoires Théa, Clermont-Ferrand, France) or Miglyol ${ }^{\circledR}$ (Laboratoires Théa) (middle right) for 48 hours, the wound surface was somewhat reduced. Incubation with $0.01 \%$ benzalkonium chloride (BAK) for 48 hours severely delayed epithelial wound healing. 


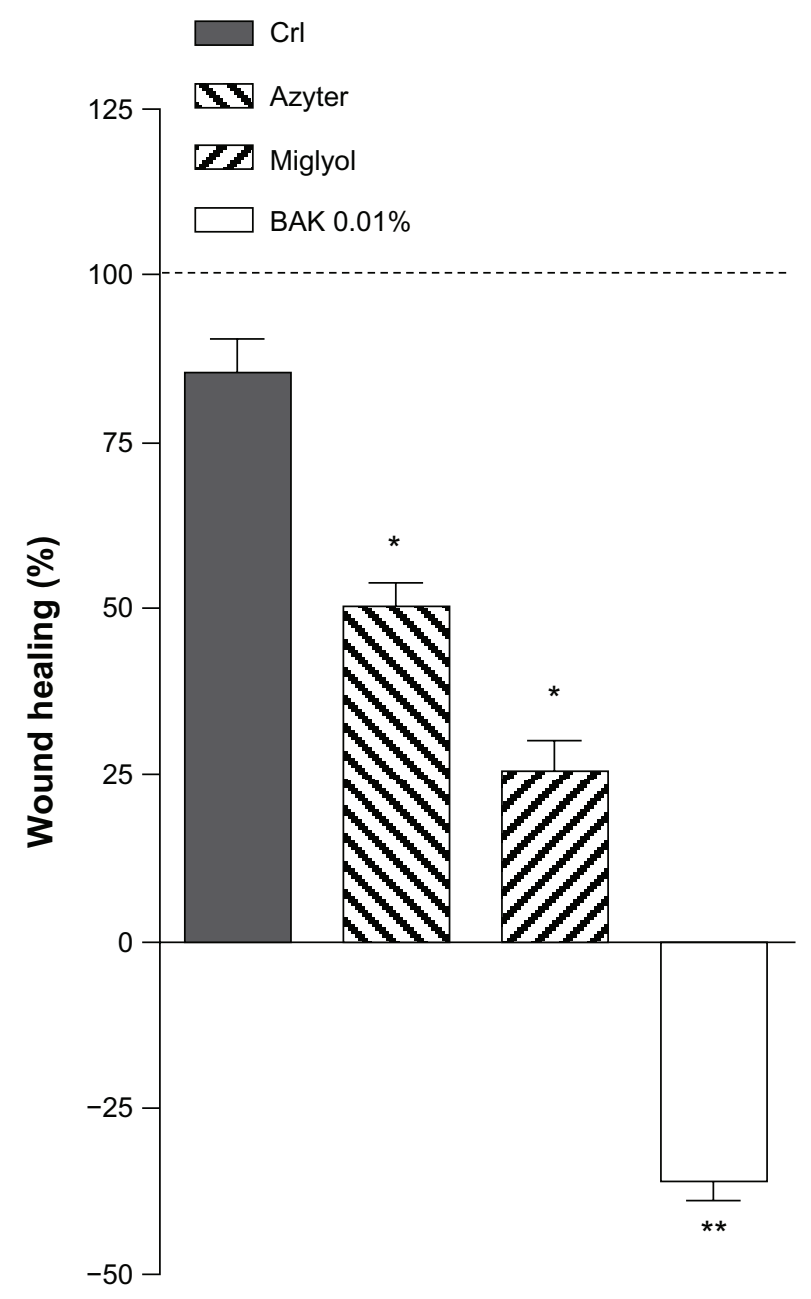

Figure 5 The wound-healing rates in Statens Serum Institut rabbit corneal (SIRC) cells incubated with different agents.

Notes: Data are expressed as percentage of healing at 48 hours as compared with wound at time $0(100 \%)$ and represent the mean \pm standard error of the mean of at least three different experiments performed in quadruplicate. Statistical analysis was performed using a one-way analysis of variance and Dunnett's post-hoc test $(* P \leq 0.05$ vs $[\mathrm{crl}] ; * * P \leq 0.01$ vs crl).

Abbreviation: BAK, benzalkonium chloride.

The current literature supports the efficacy and safety of topical azithromycin ophthalmic $1.5 \%$ solution in the treatment of various ocular surface disorders, namely bacterial conjunctivitis, blepharitis, and dry eye. Azithromycin has been shown to be efficacious against the most common bacterial pathogens of infective conjunctivitis (ie, Staphylococcus aureus, Haemophilus influenzae, Streptococcus pneumoniae $)^{23}$ and is indicated for the treatment of bacterial conjunctivitis, with the recommended dosing regimen being one drop in the affected eye twice daily ( $8-12$ hours apart) for the first 2 days, then one drop once daily for the next 5 days.

The combination of anti-inflammatory properties along with high and prolonged tissue concentrations, particularly in the lid margin and ocular surface, suggests that an azithromycin ophthalmic solution may serve as a treatment option for patients experiencing a wide range of conditions associated with the lid margin and ocular surface. ${ }^{1}$ The treatment of trachoma with topical azithromycin in addition to an oral dose is also encouraging. ${ }^{14}$

In the present study, we incubated corneal epithelial cells for 15 minutes or 6 hours with preservative-free netilmicin or levofloxacin and different concentrations of unpreserved azithromycin. Qualitative analysis was undertaken using phase-contrast optics and examination of the morphological aspects of the cell cultures. Quantitative analysis was performed by measuring the release of cytoplasmic enzyme LDH into the medium immediately and 24 hours after exposure to the study drugs. We also studied the wound-healing rates of mechanically injured corneal epithelial cells cultured for 48 hours. According to our results, unpreserved preparation of azithromycin up to a concentration of $1.5 \%$ showed a low cell toxicity, which was not significantly different from the other antibiotic preparations $(P>0.05)$, representative of the major antibiotic families of aminoglycosides and fluoroquinolones and available in preservative-free formulations.

Moreover, azithromycin did not inhibit the wound-healing process after the mechanical injury. The unpreserved preparation of azithromycin was virtually devoid of harmful effects under our experimental conditions, up to a concentration of $1.5 \%$ and also showed good recovery properties after a mechanical wound test. In control SIRC cell cultures ( $\mathrm{NaCl} 0.9 \%$ ), qualitative analysis of the corneal wound surface demonstrated that the wound was almost completely covered and repaired after 48 hours. Treatment with Azyter or Miglyol for 48 hours slightly reduced cell wound healing, whereas incubation with $0.01 \%$ BAK for 48 hours severely delayed epithelial wound healing. This evidence supports our hypothesis concerning the cytotoxic role of preservatives in commercial preparations of eye drops and the relative non-toxicity of mono-dose unpreserved antibiotics already demonstrated by a previous study. ${ }^{24}$ The low toxic effects of azithromycin may work synergistically with the previously described anti-inflammatory effects on the ocular surface.

\section{Conclusion}

This cell-culture study provides remarkable information about the in vitro toxicity profile of an unpreserved 1.5\% azithromycin ophthalmic solution. Even though this study has the limitations of an in vitro analysis, the available clinical data on tolerability appear in line with our results.

\section{Disclosure}

The authors declare no conflicts of interest in this work. 


\section{References}

1. Bremond-Gignac D, Chiambretta F, Milazzo S. A European perspective on topical ophthalmic antibiotics: current and evolving options. Ophthalmol Eye Dis. 2011;3:29-43.

2. Retsema J, Girard A, Schelkly W, et al. Spectrum and mode of action of azithromycin (CP-62,993), a new 15-membered-ring macrolide with improved potency against gram-negative organisms. Antimicrob Agents Chemother. 1987;31(12):1939-1947.

3. Amsden GW. Advanced-generation macrolides: tissue-directed antibiotics. Int J Antimicrob Agents. 2001;18 Suppl 1:S11-S15.

4. Gladue RP, Snider ME. Intracellular accumulation of azithromycin by cultured human fibroblasts. Antimicrob Agents Chemother. 1999;34(6): 1056-1060.

5. Gladue RP, Bright GM, Isaacson RE, Newborg MF. In vitro and in vivo uptake of azithromycin (CP-62,993) by phagocytic cells: possible mechanism of delivery and release at sites of infection. Antimicrob Agents Chemother. 1989;33(3):277-282.

6. Liu P, Allaudeen H, Chandra R, et al. Comparative pharmacokinetics of azithromycin in serum and white blood cells of healthy subjects receiving a single-dose extended-release regimen versus a 3-day immediaterelease regimen. Antimicrob Agents Chemother. 2007;51(1):103-109.

7. Schentang JJ, Ballow CH. Tissue-directed pharmacokinetics. Am JMed. 1991;91(3A):5S-11S.

8. Amsden GW. Anti-inflammatory effects of macrolides - an underappreciated benefit in the treatment of community-acquired respiratory tract infections and chronic inflammatory pulmonary conditions? $J$ Antimicrob Chemother. 2005;55(1):10-21

9. Ianaro A, Ialenti A, Maffia P, et al. Anti-inflammatory activity of macrolide antibiotics. J Pharmacol Exp Ther. 2000;292(1):156-163.

10. Fernandez-Robredo P, Recalde S, Moreno-Orduña M, García-García L, Zarranz-Ventura J, García-Layana A. Azithromycin reduces inflammation in a rat model of acute conjunctivitis. Mol Vis. 2013;19: 153-165.

11. Hoffmann N, Lee B, Hentzer M, et al. Azithromycin blocks quorum sensing and alginate polymer formation and increases the sensitivity to serum and stationary-growth-phase killing of Pseudomonas aeruginosa and attenuates chronic P. aeruginosa lung infection in $\mathrm{Cftr}(-/-)$ mice. Antimicrob Agents Chemother. 2007;51(10):3677-3687.

12. Howe RA, Spencer RC. Macrolides for the treatment of Pseudomonas aeruginosa infections? J Antimicrob Chemother. 1997;40(2): $153-155$

13. Bremond-Gignac D, Mariani-Kurkdjian P, Beresniak A, et al. Efficacy and safety of azithromycin $1.5 \%$ eye drops for purulent bacterial conjunctivitis in pediatric patients. Pediatr Infect Dis J. 2010;29(3): 222-226.
14. Cochereau I, Goldschmidt P, Goepogui A, et al. Efficacy and safety of short duration azithromycin eye drops versus azithromycin single oral dose for the treatment of trachoma in children: a randomised, controlled, double-masked clinical trial. Br J Ophthalmol. 2007;91(5): 667-672.

15. Cochereau I, Meddeb-Ouertani A, Khairallah M, et al. 3-day treatment with azithromycin $1.5 \%$ eye drops versus 7 -day treatment with tobramycin $0.3 \%$ for purulent bacterial conjunctivitis: multicentre, randomised and controlled trial in adults and children. Br J Ophthalmol. 2007;91(4):465-469.

16. Garnock-Jones KP. Azithromycin 1.5\% ophthalmic solution: in purulent bacterial or trachomatous conjunctivitis. Drugs. 2012;72(3): 361-373.

17. Wingard JB, Romanowski EG, Kowalski RP, et al. A novel cell-associated protection assay demonstrates the ability of certain antibiotics to protect ocular surface cell lines from subsequent clinical Staphylococcus aureus challenge. Antimicrob Agents Chemother. 2011;55(8):3788-3794.

18. Valente C, Lester M. Impact of glaucoma medication on ocular tissue. Expert Rev Ophthalmol. 2010;5(3):405-412.

19. Seitz B, Hayashi S, Wee WR, La Bree L, Mc Donnell PJ. In vitro effects of aminoglycosides and fluoroquinolones on keratocytes. Invest Ophthalmol Vis Sci. 1996;37:656-665.

20. Pellegrini-Giampietro DE, Cozzi A, Peruginelli F, et al. 1-Aminoindan1,5-dicarboxylic acid and (S)-(+)-2-(3'-carboxybicyclo[1.1.1] pentyl)glycine, two mGlu1 receptor-preferring antagonists, reduce neuronal death in in vitro and in vivo models of cerebral ischaemia. Eur $J$ Neurosci. 1999;11(10):3637-3647.

21. Kim TI, Tchah H, Lee SA, Sung K, Cho BJ, Kook MS. Apoptosis in keratocytes caused by mitomycin C. Invest Ophthalmol Vis Sci. 2003;44(5):1912-1917.

22. Kim SY, Lim JA, Choi JS, Choi EC, Joo CK. Comparison of antibiotic effect and corneal epithelial toxicity of levofloxacin and moxifloxacin in vitro. Cornea. 2007;26(6):720-725.

23. Seal DV, Barrett SP, McGill JI. Aetiology and treatment of acute bacterial infection of the external eye. Br J Ophthalmol. 1982;66(6):357-360.

24. Mencucci R, Paladini I, Pellegrini-Giampietro DE, et al. In vitro comparison of the cytotoxic effects of clinically available ophthalmic solutions of fluoroquinolones on human keratocytes. Can JOphthalmol. 2011;46(6):513-520.
Clinical Ophthalmology

\section{Publish your work in this journal}

Clinical Ophthalmology is an international, peer-reviewed journal covering all subspecialties within ophthalmology. Key topics include: Optometry; Visual science; Pharmacology and drug therapy in eye diseases; Basic Sciences; Primary and Secondary eye care; Patient Safety and Quality of Care Improvements. This journal is indexed on Submit your manuscript here: http://www.dovepress.com/clinical-ophthalmology-journal

\section{Dovepress}

PubMed Central and CAS, and is the official journal of The Society of Clinical Ophthalmology (SCO). The manuscript management system is completely online and includes a very quick and fair peer-review system, which is all easy to use. Visit http://www.dovepress.com/ testimonials.php to read real quotes from published authors. 